\title{
Analysis of a PM DC Motor Model for Application in Feedback Design for Electric Powered Mobility Vehicles
}

\author{
P. Wolm ${ }^{1}$, X.Q. Chen ${ }^{1}$, J.G. Chase ${ }^{1}$, W. Pettigrew ${ }^{2}$, C.E. Hann ${ }^{1}$ \\ ${ }^{1}$ Department of Mechanical Engineering, University of Canterbury \\ PO Box 4800, Christchurch 8140, New Zealand \\ Email: pwo19@student.canterbury.ac.nz, \{xaoqi.chen, geoff.chase\}@canterbury.ac.nz z \\ ${ }^{2}$ Dynamic Controls Ltd, 17 Print Place, Middleton, Christchurch 8015, New Zealand \\ Email: wpettigrew@dynamiccontrols.com
}

\begin{abstract}
Accurate modelling of permanent magnet (PM) DC motors is a prerequisite for expedient feedback design of electric powered mobility vehicles. This paper identifies the parameters in the ideal equations for PM DC motors and considers the methods used to measure and calculate them accurately. However, the ideal PM DC motor equations' outcomes are rarely accurate compared with actual results and measurements. The model inaccuracy arising from the existing theoretical model, which is often used in literature and by researchers, has been observed. The work discusses the method to increase the model accuracy, and analyse the model for the application in feedback design for a target vehicle. Tests have been conducted on the actual motors with a real-time data acquisition instrument. Results have been obtained and analysed to validate the improved PM DC model.
\end{abstract}

Index Terms - PM DC motor model, electric powered mobility vehicles, feedback design, frictional torque.

\section{INTRODUCTION}

In the design of electric powered mobility vehicles used for medical purposes, high safety standards must be met. In order to design, verify and tune systems such as feedback controls that can be used for a variety of different types of mobility vehicles, an accurate model of such vehicles would expedite the process and aid greatly in reaching or exceeding safety requirements. Although such models would comprise both physical dynamics and electrical dimensions, the electrical drivers which are normally PM DC motors would be an initial starting point for accurate model development. Quite often data plate values are incomplete or missing entirely. Confirmation of any values provided is a must for any model or contiguous batch of PM DC motors being used particularly as the drivers are usually geared and treated as a single system which has an effect on parameters. Once the motor-system parameters are known each control system is closer to being made unique to each wheelchair, scooter, etc.

Many openly available sources of relevant engineering texts [1-3] and academic papers [4-6] list the basic parameters for DC motors but leave it to the reader to intuitively ascertain that the listed parameters apply only to an ideal model. Of particular concern is the treatment of friction within a DC motor system. Rizzoni [1] states "friction losses in the load are represented by the viscous friction coefficient", implying that no other friction is considered or exists. Meanwhile, Westphal [2] goes farther stating "The only appreciable friction effect in operation is viscous friction and coulomb and static frictions are neglected" giving a hint that there are other possible parameters to be considered. Other sources mention various other parameters such as $T_{f}$, frictional torque [7] which is not fully expanded upon.

This work presents an accurate model of an actual geared PM DC motor and the difference in the respective results between the ideal theoretical equations and a model with an enhanced equation to actual measurements. Firstly the ideal equations and parameters and the methods of measuring and calculating the parameters are discussed. Then the results of the ideal model are contrasted against actual values. This is followed by discussion of the enhanced equation with an additional parameter including its method of calculation. Actual results are then compared to the outputs of a Simulink model of the geared PM DC motor with the enhanced equation.

\section{PM DC MOTOR MODEL}

\section{A. Ideal Equations}

The equivalent circuit model for an ideal DC motor is depicted in Figure 1.

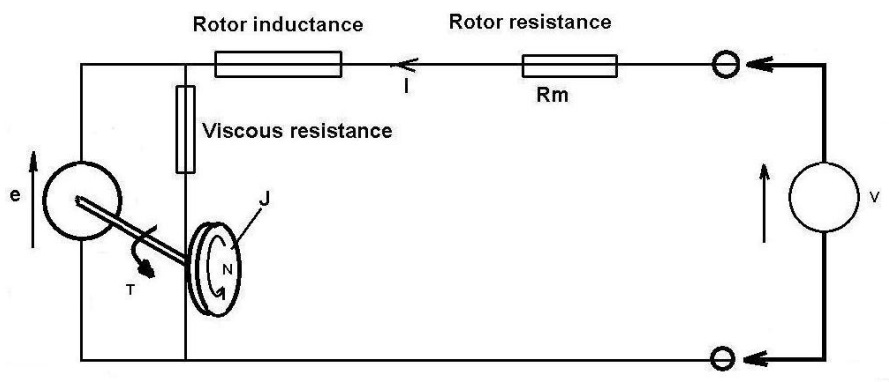

Figure 1: Ideal DC motor model. 
Using Kirchoff's voltage law and summing the voltages throughout a simple circuit of a DC motor drive system results in

$$
V=R_{a} i+L_{a} \frac{d i}{d t}+V_{e m f}
$$

where $R_{a}, L_{a}$ and $V_{e m f}$ are the armature (or rotor) resistance, armature inductance and the back emf.

The equation of motion for a DC motor drive, ignoring any load, gives torque as a second order ODE in rotation as

$$
T=\ddot{\theta}+B \dot{\theta}
$$

where $J$ and $B$ are the motor inertia (or equivalent system inertia in a geared motor system reflected on the motor shaft) and the viscous friction coefficient, also known as viscous damping coefficient (or equivalent viscous friction for a geared motor system).

The electrical and mechanical components are coupled in two ways. First, an approximate relation generally describes motor torque as a linear function of current in the motor:

$$
T=k_{t} i
$$

where $k_{t}$ is the motor torque constant. Secondly, the back emf in the motor is linearly related to the motor rotational velocity,

$$
V_{e m f}=k_{e} \dot{\theta}
$$

where $k_{e}$ is variously known as the speed, electrical, motor or back emf constant. The combined electrical and dynamic relationships result in a system of equations that govern a DC motor system's response. Equations (3) and (4) are substituted into (2) and (1) respectively to result in the final system equations of an ideal PM DC motor model; a 1st and 2nd order ODE that can provide two results, $i$ and $\theta$ :

$$
\begin{gathered}
k_{t} i=J \ddot{\theta}+B \dot{\theta} \\
V=R_{a} i+L_{a} \frac{d i}{d t}+k_{e} \dot{\theta}
\end{gathered}
$$

Representing (5) and (6) in a model of state space form provides

$$
\begin{gathered}
{\left[\begin{array}{c}
\ddot{\theta} \\
\frac{d i}{d t}
\end{array}\right]=\left[\begin{array}{cc}
\frac{-B}{J} & \frac{k_{t}}{J} \\
\frac{-k_{e}}{L_{a}} & \frac{-R_{a}}{L_{a}}
\end{array}\right]\left[\begin{array}{l}
\dot{\theta} \\
i
\end{array}\right]+\left[\begin{array}{c}
0 \\
\frac{1}{L_{a}}
\end{array}\right] V} \\
\dot{\theta}=\left[\begin{array}{ll}
1 & 0
\end{array}\right]\left[\begin{array}{c}
\dot{\theta} \\
i
\end{array}\right]
\end{gathered}
$$

\section{B. Measurement and Calculation of Parameters}

Torque Constant $\boldsymbol{k}_{\boldsymbol{t}}$ : Rearranging (3) provides the torque constant in terms of torque versus current. Hence, this requires concurrent torque and current measurements.

Figure 2 shows the test set-up for torque measurement. It consists of a geared PM DC motor with wheel rim, two Newton force meters, a digital multi-meter (DMM) to measure voltage at the motor terminals, a DMM clamp meter to measure current, and a rope to provide a load on the motor.

A steady $24 \mathrm{~V}$ was input, and the motor was allowed to reach a constant speed. The load was steadily increased until motor stall. Motor current, the forces on both ends of the rope, and motor terminal voltage to ensure that voltage was steady at the motor terminals were measured and recorded at set intervals. The motor torque was calculated by multiplying the difference between the two forces on each end of the rope with the radial distance between wheel hub and where the rope rested on the wheel rim, i.e. $T=F r$.

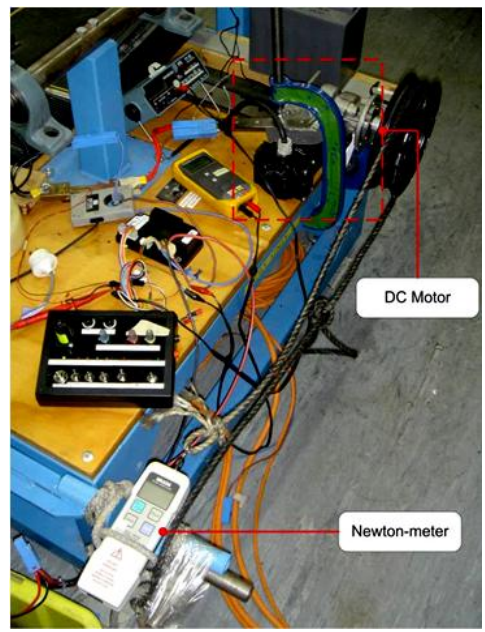

Figure 2: Test rig to calculate torque constant

Figure 3 shows the results with the slope of the line providing the torque constant; $1.4882 \mathrm{Nm} / \mathrm{A}$.

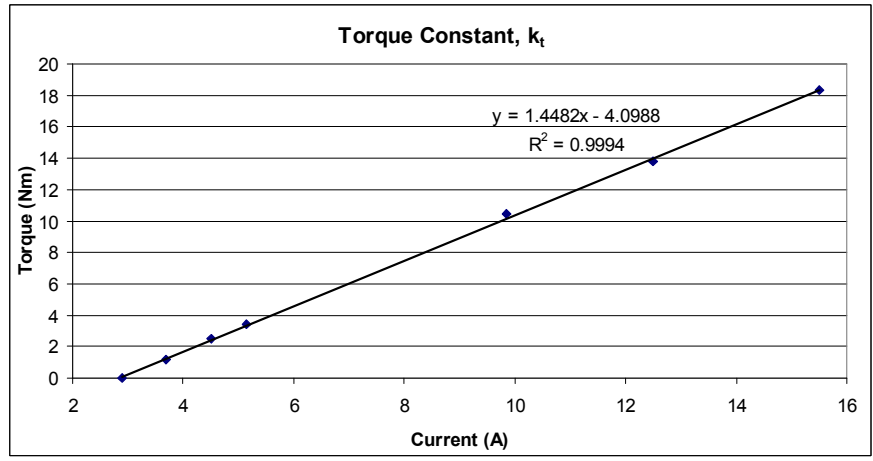

Figure 3: Results of torque versus current test

Speed Constant, $\boldsymbol{k}_{\boldsymbol{e}}$ : If only looking at steady state values, rearranging (6) to give slope-intercept form results in 


$$
\frac{V}{i}=k_{e}\left(\frac{\dot{\theta}}{i}\right)+R_{a}
$$

where $\dot{\theta}$ is the motor shaft rotational speed and not speed of the gear output shaft. From this only steady state values of input voltage, current and motor shaft velocity need to be measured. The test set-up included the same geared PM DC motor with wheel, a steady $24 \mathrm{~V}$ supply, DMM to measure terminal voltage, DMM clamp meter to measure current, a means to control motor velocity, i.e. voltage at the motor terminals, which in this case was a variable resistance, and a digital rpm gauge that measured the rpm of the wheel which was taken to equal the motor shaft velocity. Figure 4 provides the results of measurements made at multiple terminal voltage inputs under "no load" conditions with $k_{e}$ equalling the slope of the resulting line; $1.685 \mathrm{rad} / \mathrm{s} / \mathrm{V}$.

It should be noted that in an ideal DC motor, the speed constant is equal to the torque constant. The measurements obtained by experiment indicate this is not the reality.

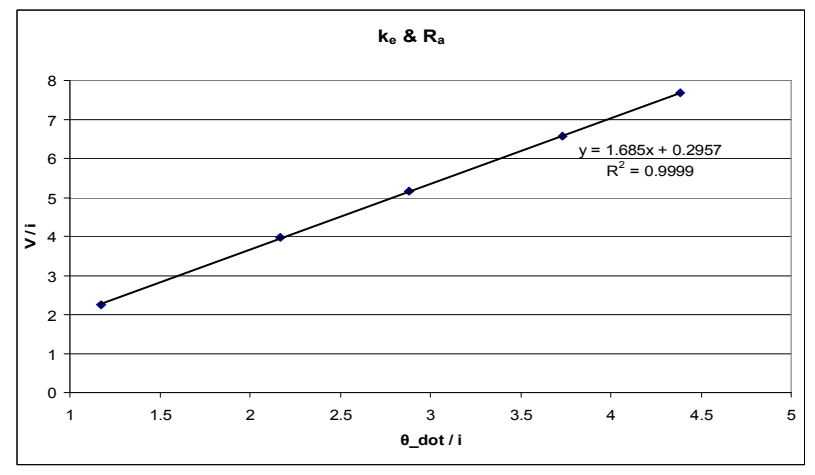

Figure 4: Steady state results of (9).

Armature Resistance, $\boldsymbol{R}_{\boldsymbol{a}}$ : By utilising (9) and the results for the speed constant, armature resistance can be taken from the equation of the line. It is the y axis intercept value, 0.2957 ohms $(\Omega)$, as seen in Figure 4 .

Viscous Friction Coefficient, $\boldsymbol{B}$ : Under steady state conditions (5) becomes

$$
k_{t} i=B \dot{\theta}
$$

Rearranging (10) provides

$$
B=k_{t} i / \dot{\theta} \text { or } B=k_{t} i / \omega
$$

A range of steady state current and corresponding rotational velocity values were measured with the same set-up as used to calculate the speed constant. The range in values was used to calculate $B$ such that $i / \omega$ became $\Delta i / \Delta \omega$, with $k_{t}$ already known. The friction coefficient also takes into account the gearing and eddy current losses in the motor iron which increase with speed. Hence $B$ tends to be a 'lumped' term.

Armature Inductance, $\boldsymbol{L}_{\boldsymbol{a}}$ : To measure the armature inductance a digital meter capable of measuring and recording instantaneous or real-time changes was needed. The instrument used was a Fluke 105B Scopemeter. The test set-up of the geared PM DC motor also required a steady dc voltage supply. A fixed voltage was supplied to the at-rest motor system, with the rotor/wheel locked. The rate of current rise with time was captured by the meter and inductance was calculated using (12) following this paragraph. Back emf is zero since there is no rotor rotation. Initial current is zero and subsequently negligible when compared with rate of change of current within the first instance of motor start-up. As a result (1) becomes, when rearranged

$$
L_{a}=V /\left(\frac{d i}{d t}\right)
$$

Within the first $3 \mathrm{~ms}$ the current went from 0 to 44 A with a 12 V step input resulting in $L_{a}=0.82 \mathrm{mH}$.

Motor/System Inertia, $\boldsymbol{J}$ : Once again the real-time measuring meter, the Fluke 105B Scopemeter was needed and used the same test set-up as for armature inductance but with a means to control voltage input and hence motor current levels. In this case the rate of voltage change needed to be measured. The motor rotor was held locked until a set current level was reached, overcoming the initial effects of rotor inductance, and then released. Once again the rotational velocity is initially zero and subsequently negligible when compared with the rate of rotational acceleration within the first instance of motor release. To calculate motor/system inertia (5) was rearranged taking into account negligible initial velocity to give

$$
J=\left(k_{t} i\right) / \ddot{\theta}
$$

The rotational acceleration was initially in units of $\mathrm{V} / \mathrm{s}$ but was then multiplied by the speed constant to convert to units of $\mathrm{rad} / \mathrm{s}^{2}$. With a steady release current of $17.2 \mathrm{~A}$ the resulting system inertia was $0.271 \mathrm{~kg} \cdot \mathrm{m}^{2}$.

\section{GEARED PM DC MOTOR}

\section{A. Measured Outputs}

The relevant measured outputs have been mentioned under II. $B$ for the speed constant where steady state values of voltage input, motor current and output angular velocity of the motor shaft were recorded. In particular, the velocity of the rotating wheel was taken to be equal to the rotational velocity of the of the geared PM DC motor shaft. This is a result of 'lumping' the moment of inertia of the motor rotor with the gearing and wheel rim including the tire.

Figure 5 indicates the actual relevant steady state measurements over an arbitrary time period, hence the areas of no data between steady state values. 


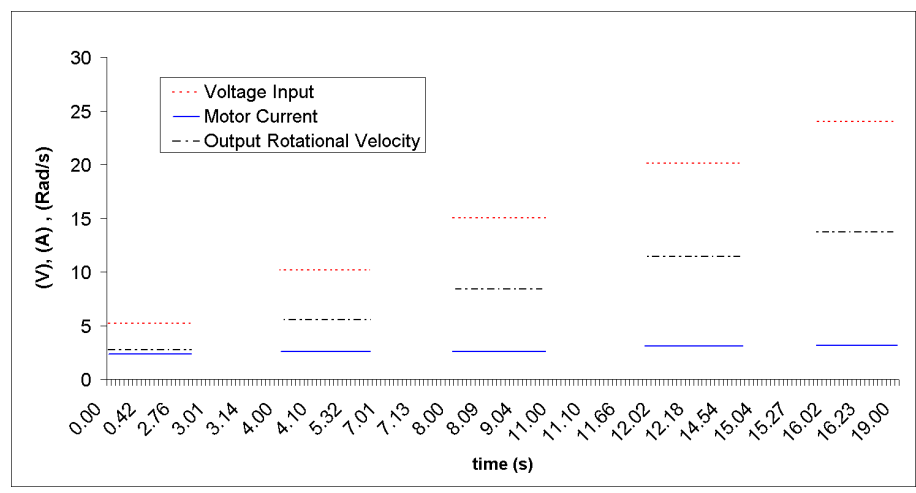

Figure 5: Measured steady state values of PM DC geared motor.

It can be seen that the output rotational velocity increases as expected as does the motor current although very slightly, also as expected. This is predicted in (1) where under higher rotational velocities the back emf becomes predominant. Hence voltage is limited internally in the motor and therefore also motor current. Once a load is applied, back emf is decreased as the motor slows. This increases the voltage difference internally thereby increasing current flow and the torque to maintain a given rotational velocity for a given voltage input and loading.

\section{B. Measured Outputs versus Ideal Model}

As stated for the state space model of an ideal PM DC motor, the output can be in terms of rotational velocity of the output shaft/rotor or the motor current. The rotational velocity output is important in that it is used to calculate the driving forces provided by the wheels of a wheelchair or other mobility vehicles. Internal motor current can be used if necessary in feedback design for what is known as "load compensation". Thus, these are two outputs that are most relevant with regard to modelling and feedback design and which are most necessary to compare to actual values.

Figure 6 shows the measured current and the equivalent ideal model output for the same given voltage inputs.

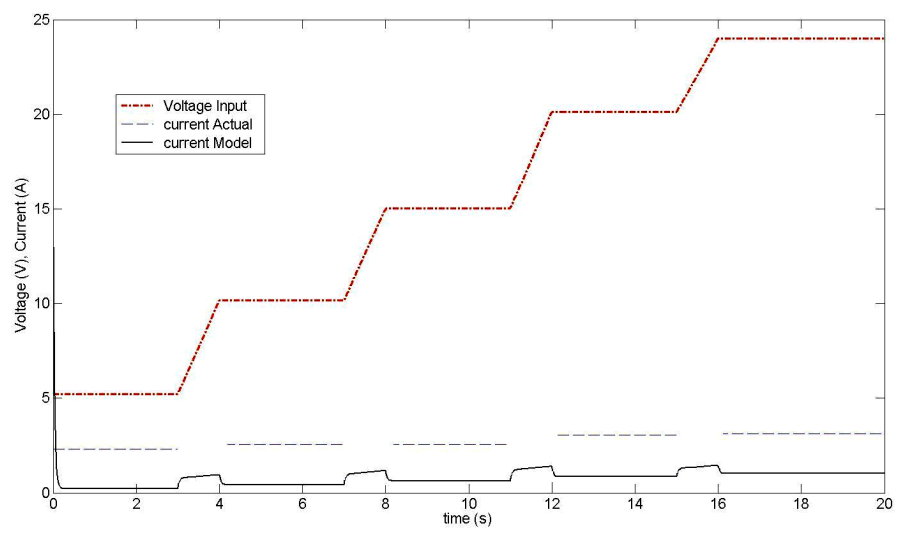

Figure 6: Actual current measured versus equivalent ideal model output.

The Simulink model has interpolated between the steady state voltage inputs to give the continuous graph of voltage.
Furthermore, the Simulink model interpreted the initial voltage entered, which was not zero, as a step. Hence, there is an initial current spike which emulates what would actually happen in a PM DC motor when a sudden step input of voltage occurs and recorded continuously.

Note that the subsequent current spikes are 'muted'. They would be similar to the initial current spike. The muting is due to the relatively shallow slope of the voltage rise between inputs due to Simulink's solver interpolation. However the intent is accurate as are the steady state values for the given parameters. Most notable is the large difference between the actual current measured and the ideal theoretical model's equivalent output.

Figure 7 provides contrast between the measured rotational velocity and the equivalent output of the motor model for given input voltages.

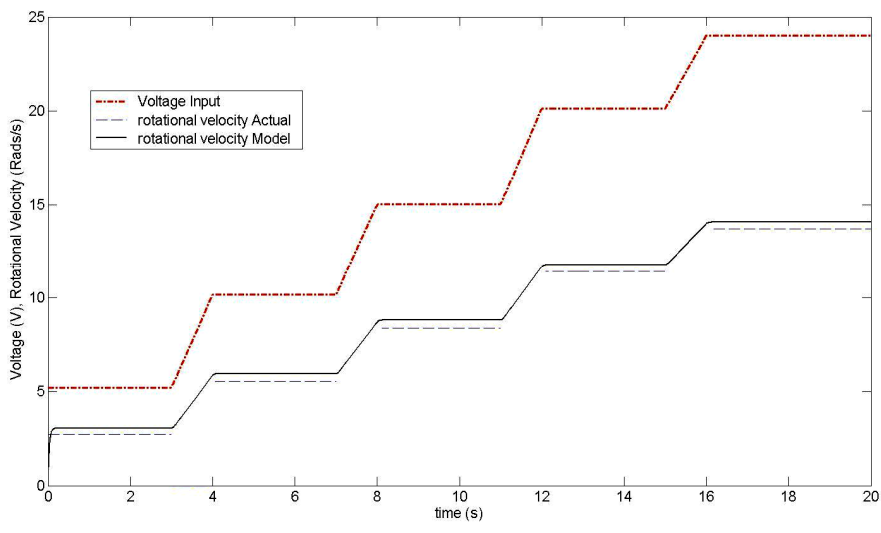

Figure 7: Actual rotational velocity measured versus equivalent ideal model output.

It is clear that the model output of rotational velocity is much closer to the actual measurements. The initial increase and gradual levelling of the model's rotational velocity (as seen to the far left of the Figure 7) is also in keeping with what would be seen of an actual PM DC motor with voltage step inputs. The subsequent changes are the same as for the subsequent changes given in the current comparison of Figure 6.

\section{ENHANCED PM DC MOTOR MODEL EQUATION}

\section{A. Additional Parameter}

As stated in the introduction there have been hints of additional sources of friction. Jang and Lee [8] make mention of DC motor friction modelling and in particular the Coulomb friction model and mention frictional torque as do Rahman and Hoque [7]. However neither makes the connection between Coulomb friction and frictional torque and how they may be identified. Kara and Eker [9] discussed Coulomb friction using the same symbology as for frictional torque and Sepp [10] goes on to clearly lay out the friction model including viscous, Coulomb and stiction or static friction.

Coulomb friction is considered to be a constant retarding force but is discontinuous over zero crossings. That is, when a 
motor reverses direction it must come to a stop at which point Coulomb friction drops to zero and then opposes the reversed direction. In effect Coulomb friction is constant when rotational velocity is not zero. Both Kara and Eker and Sepp consider Coulomb friction as a non-linear effect, although Kara and Eker state "the linear model is capable of supplying the required robustness property when the mechanical system rotates in a single direction".

In the case of modelling for feedback control of wheelchairs or other mobility vehicles, dynamic effects such as over or under-steer are noticeable under motion and the higher the velocities, the more noticeable. In these cases Coulomb friction can be treated as a linear constant. Any non-linearity associated, and stiction as well, would only be a factor with stop-start dynamics such as 'jerk' motion. For the purposes of this paper Coulomb friction is treated as linear. The enhanced DC motor model is shown in Figure 8.

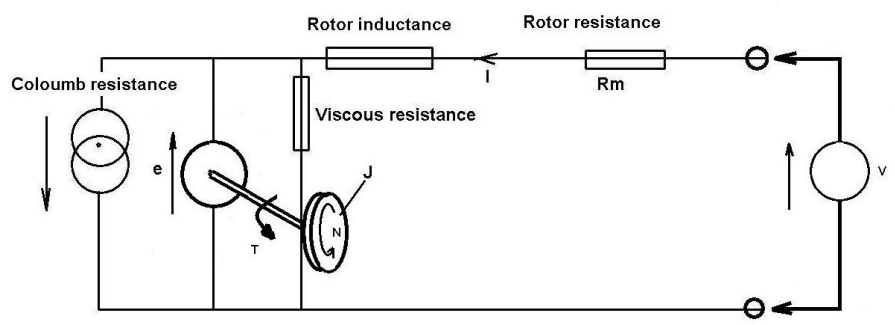

Figure 8: Enhanced DC motor model.

Through the friction model, now recognising Coulomb friction, $\tau_{\mathrm{f}}$, (2) becomes

$$
T=\ddot{\theta}+B \dot{\theta}+\tau_{\mathrm{f}}
$$

Under steady state conditions, substituting in (3) and rearranging provides Coulomb friction in units of $\mathrm{Nm}$.

$$
\tau_{\mathrm{f}}=k_{t} i-B \dot{\theta}
$$

The same series of measurements as were used to calculate the speed constant were the values of input into (15), namely current and rotational velocity. Both the torque constant and viscous friction coefficient had been calculated previously. The average of the series of calculations provided the Coulomb friction value.

\section{B. Measured Outputs versus Enhanced Model}

Figure 9 and Figure 10 compare the actual measured values for current and rotational velocity respectively with the equivalent enhanced model outputs for given voltage inputs.

As can be seen in both Figure 9 and Figure 10 the model outputs closely follow both actual measured values for current and rotational velocity.

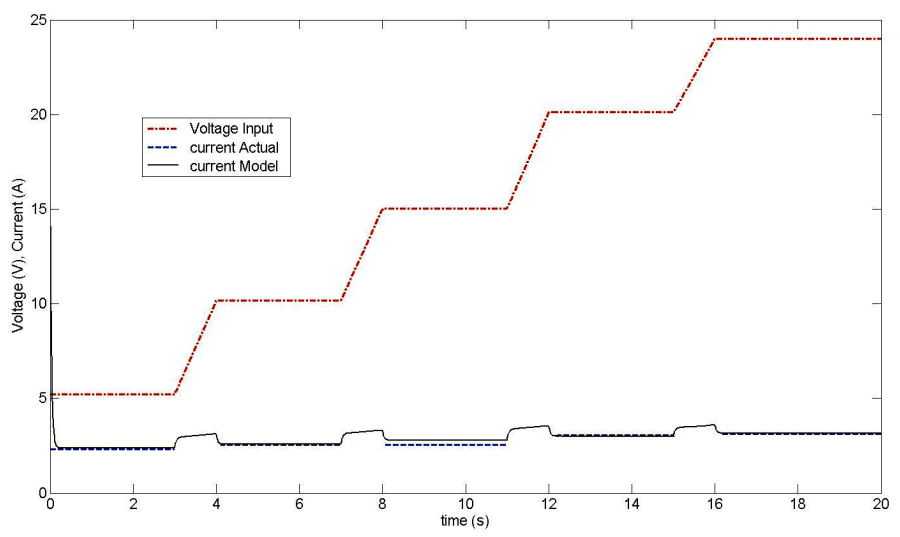

Figure 9: Actual current measured versus equivalent enhanced model output

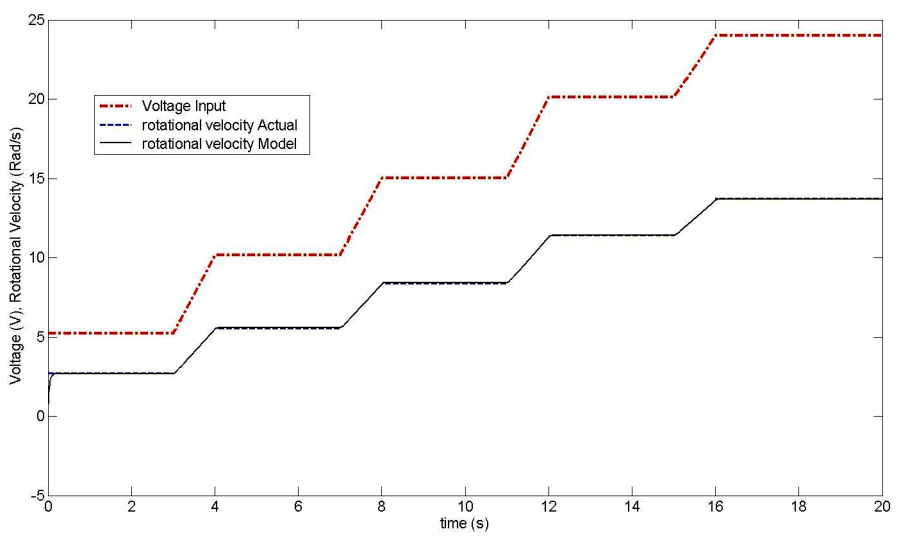

Figure 10: Actual rotational velocity measured versus equivalent enhanced model output

Table 1 provides the results of mean square error (MSE) method as a means to validate the enhanced PM DC motor model using the steady state values found in Figures 7 and 8.

Table 1 measurement results of current and rotational velocity.

\begin{tabular}{|c|c|c|c|c|c|}
\hline $\begin{array}{c}\text { Input } \\
\text { Voltage (V) }\end{array}$ & 5.22 & 10.17 & 15.03 & 20.11 & 24.01 \\
\hline Current (A) & 0.003 & 0.036 & 0.131 & 0.037 & 0.035 \\
\hline $\begin{array}{c}\text { Rotational } \\
\text { Velocity } \\
\text { (Rad/s) }\end{array}$ & 0.002 & 0.001 & 0.002 & 0.002 & 0.002 \\
\hline
\end{tabular}

\section{CONCLUSIONS}

The experimental test bed comprising a front wheel drive vehicle motorised by two PM DC motors, actuators/sensors, and real-time data acquisition system has been reported in [11]. This paper addresses accurate modelling for a geared PM DC motor as a first step to creating a full model for the dynamics of a wheelchair or mobility vehicle. Since the DC motors are the only source of directly controllable driving force in the above mentioned vehicles, it is essential that they be accurate.

The comparison of ideal PM DC motor model output and experimental results shows errors in applying the standard model to the geared PM DC motor. This work introduced Coulomb friction torque as an additional parameter. The 
improved model is more accurate, and has been validated by experiments. There is a close agreement between the model output and the measurement data.

By creating the accurate motor model a crucial stage in practical control problems for wheelchairs and mobility vehicles has been set. With the above solid basis, an adequate dynamic system model can be developed to test the reliability of designed control.

\section{ACKNOWLEDGEMENT}

This work is supported by the research grant DCLX0601 from Foundation of Research, Science and Technology (FRST). The authors would like to thank the support and technical inputs from Dynamic Controls Limited.

\section{REFERENCES}

[1] Rizzoni, G. (2004) Principles and Applications of Electrical Engineering, McGraw-Hill, New York, pp. 869 \& 875.

[2] Westphal, L.C. (2001) Handbook on Control Systems Engineering, Springer, pp. 174-176.

[3] Attinyas, Y. (2000) Manufacturing Automation: Metal Cutting Mechanics, Machine Tool Vibrations, and CNC Design, Cambridge University Press, pp. 167.

[4] Chen JX, Guo YG, Zhou JG, Jin JX, "Performance Analysis of a Surface Mounted Permanent Magnet Brushless DC Motor using an Improved Phase Variable Model", Record of the 2007 IEEE Industry Application Conference, pp. $2169-2174$.

[5] Kim CG, Lee JH, Kim HW, Youn MJ, "Study on Maximum Torque Generation for Sensorless Controlled Brushless DC Motor with Trapezoidal Back EMF", IEE Proceedings Electric Power Applications, Vol 152, No 2, March 2005, pp. 277 - 291.

[6] Haji H, Toliyat HA, "Rotor Eccentricity Fault Detection of a DC Motor", Proceedings of $27^{\text {th }}$ Annual Conference of IEE, Industrial Electronics Society, Vol 1, 2001, pp. 591-596.

[7] Rahman MA, Hoque MA, "On-Line Self-Tuning ANN-Based Speed Control of a PM DC Motor" IEEE/ASME Transactions on Mechatronics", Vol 2, No 3, Sep 1997, pp. 169 - 178.

[8] Jang JO, LEE PG, "Neuro-fuzzy Control for DC Motor Friction Compensation", Proceedings of the 39th IEEE Conference on Decision and Control, Vol 4, 2000 , pp. 3550 - 3555.

[9] Kara T, Eker I, "Nonlinear Modelling and Identification of a DC Motor for Bidirectional Operation with Real Time Experiments", Journal of Energy Conversion and Management, Vol 45, 2004, pp. 1087-1106.

[10] Sepp K, "Friction Modelling in Linear Track Cart Pendulum System", [www.aa.washington.edu/courses/aa449/frictionmod99.pdf.], May 2008, unpublished.

[11] Chen XQ, Wolm P, Elliot R, Chase JG, Pettigrew W, “A Wireless Testbed of Front Wheel Drive Wheelchair for Stability Control Prototyping", Conference Proceedings Computational Intelligence Robotics and Autonomous Systems, ISBN 978-0-473-12433-5, pp. 261266, Palmerston North, New Zealand, 28th - 30th November, 2007. 